\title{
Bubble-size distributions produced by wall injection of air into flowing freshwater, saltwater and surfactant solutions
}

\author{
Eric S. Winkel, Steven L. Ceccio, David R. Dowling, Marc Perlin
}

\begin{abstract}
As air is injected into a flowing liquid, the resultant bubble characteristics depend on the properties of the injector, near-wall flow, and flowing liquid. Previous research has shown that near-wall bubbles can significantly reduce skin-friction drag. Air was injected into the turbulent boundary layer on a test section wall of a water tunnel containing various concentrations of salt and surfactant (Triton-X-100, Union Carbide). Photographic records show that the mean bubble diameter decreased monotonically with increasing salt and surfactant concentrations. Here, 33 ppt saltwater bubbles had one quarter, and $20 \mathrm{ppm}$ Triton-X-100 bubbles had one half of the mean diameter of freshwater bubbles.
\end{abstract}

\section{1}

\section{Introduction}

Turbulent boundary layer (TBL) skin-friction in liquid flows can be reduced by the injection of air bubbles from the wall on which the boundary layer forms. Experimental results from several laboratories (Bogdevich and Evseev 1976; Madavan et al. 1984a; Meng and Lovett 1998) show that TBL skin-friction decreases with increasing near-wall void fraction and that peak skin-friction reductions of $80 \%$ or more are possible. At present, the mechanism(s) by which bubbles influence skin-friction is (are) not fully understood, although buoyancy, bubble deformation, bubble-turbulence interactions, increased effective viscosity, and simple density reduction have been identified as potentially important phenomena (Lumley 1984; Legner 1984; Kato et al. 1993; Kitagawa et al. 2003; Kunz et al. 2003). A prevalent notion is that bubbles must change, influence, or otherwise disrupt momentum

Received: 12 December 2003 / Accepted: 23 June 2004

Published online: 20 October 2004

(C) Springer-Verlag 2004

E.S. Winkel ( $ه)$, S.L. Ceccio, D.R. Dowling

University of Michigan, Mechanical Engineering,

Ann Arbor, MI, USA

E-mail: ewinkel@engin.umich.edu

M. Perlin

University of Michigan,

Naval Architecture and Marine Engineering,

Ann Arbor, MI, USA

The authors of this paper are grateful for the support of the Office of Naval Research under contract N00014-02-C-0468, Dr. L. P. Purtell, technical monitor. transfer in the TBL's buffer layer to reduce skin-friction (Merkle and Deutsch 1992).

Although the understanding of bubble-based TBL skin-friction drag reduction is incomplete, the importance of bubble size can be readily ascertained by considering the ratio $d^{+}=d / l_{v}$ of bubble diameter, $d$, to the local boundary-layer viscous wall unit, $l v=v / \sqrt{\tau_{\mathrm{w}} / \rho}$ where $v$ is the fluid kinematic viscosity, $\tau_{\mathrm{w}}$ is the wall shear stress, and $\rho$ is the fluid mass density. This ratio compared to the vertical distance from the wall to the buffer layer $\left(5<y^{+}<30\right.$, where $y$ is the wall normal coordinate and $y^{+}=y /$ $l_{v}$ ) yields important information. If the bubbles in the buffer layer are smaller than or comparable to a wall unit, then the bubbles might merely act to reduce the water density because they are small compared to the size of an overturning eddy. On the other hand, if the bubbles are much larger than $30 l_{v}$, it may not be possible for them to alter the fluid momentum exchange in the buffer layer unless they are present in sufficient number to substantially increase the buffer layer thickness.

Recent experimental studies in low-Re TBL flows (Kawamura et al. 2003) confirm the importance of bubble size (and number density) in skin-friction drag reduction. Kawamura et al. produced bubbles in two different size ranges using different injection methods. First, air was injected through a porous plate to produce bubbles in the range of $0.5-2.0 \mathrm{~mm}$. In the second method, water saturated with dissolved air was injected through a slot after a $0.7 \mathrm{MPa}$ pressure drop. Here, the dissolved air foamed downstream of the slot to create much smaller bubbles with diameters of order $0.1-0.01 \mathrm{~mm}$. They report that the smaller bubbles produced by foaming dissolved air could be up to twice as effective for drag reduction as the larger bubbles for equal void fractions. Using photographic techniques, they report that the smaller bubbles remain closer to the wall than those generated by forcing air through a porous metal plate. In contrast, a separate study involving a $50 \mathrm{~m}$ flat towed body has reported no apparent difference in skin-friction drag when employing a surfactant to reduce bubble size (Takahashi et al. 2001).

The results in this paper show that when air is injected into a TBL the bubble-size distribution depends on the concentration of salt or surfactants in the flowing water. To date, nearly all bubble drag reduction experiments have been performed in laboratory water tunnels with freshwater; whereas, commercial or military implementations of bubble drag reduction would most likely occur in ocean water containing natural salts and surfactants. The purpose of this paper is to document the changes in 
bubble-size distributions observed downstream of a flush-mounted porous-plate air injector when salinity and surfactant concentrations are varied between freshwater and seawater levels. In particular, the results presented here show that bubble sizes are much smaller in saltwater than in freshwater. Therefore, previous bubble drag reduction experiments in freshwater may not accurately predict bubble drag reduction in seawater. In the context of drag reduction applications, Meng and Uhlman (1998) discuss the effects of smaller bubbles in seawater and the likely physical mechanisms responsible for this size reduction.

Previous research has shown that different aqueous solutions have significant effects on both the formation and coalescence of air bubbles. In a bubble riser column, Zieminski and Whittemore (1971) measured the bubblesize distributions of air injected through a porous plate into eleven different electrolyte solutions. They found that the interfacial surface area of the injected air increased linearly (indicating a decrease in bubble size) with ionic strength for all eleven solutions tested. In a similar experiment, Keitel and Onken (1982) reported that the Sauter mean diameter of air injected through a porous plate decreases monotonically with increasing ionic strength. They also report a critical molar concentration above which coalescence inhibition was significant, and that the critical concentration was proportional to the size of the hydrated ions. These two bubble riser experiments illustrate that bubble-size distributions are affected directly by ionic strength. However, because the bubbles were measured at a significant distance from the injector, $1.5 \mathrm{~m}$ for Keitel and Onken (1982), it is unclear whether the reduction in bubble size is a result of coalescence inhibition, altered bubble formation, or both.

In a bubble "shattering" experiment, Slauenwhite and Johnson (1999) expanded small $(5 \mu \mathrm{L})$ slugs of gas through an $86 \mu \mathrm{m}$ diameter orifice into different liquid solutions and observed the break up of the air volume. They observed that equivalent gas volumes shattered into at least four times the number of bubbles in saltwater than in freshwater. Thus it is clear that not only is bubble coalescence different in ionic solutions, but the manner in which bubbles are formed is fundamentally different in seawater and freshwater. In addition, they found similar results for bubble formation in both natural seawater and artificially prepared seawater.

Bubble-size distributions have also been measured in various aqueous solutions in turbine-stirred, aerated vessels. Machon et al. (1997) observed a critical molar concentration above which bubble coalescence was inhibited and significantly decreased bubble sizes were

observed. Their results also showed that there was no direct relationship between liquid surface tension and bubble size. In a similar experiment, Alves et al. (2002) measured bubble diameters throughout the entire volume using pure water and electrolyte and surfactant solutions. In all three cases, bubble size decreased monotonically with turbine power input, and the electrolyte and surfactant solutions showed significantly more decrease than did pure water. This result is analogous with the conclusions of Merkle and Deutsch (1992) that drag reduction applications exhibit a bubble diameter decrease with increasing free-stream speed.

To date, the mechanisms responsible for bubble formation, size distributions, and coalescence in saltwater (and other ionic solutions) are not completely understood. The presence of an electrical double layer, where there is a net electrostatic repulsion between surfaces, strongly supports the observed decrease in bubble diameter with ionic strength. Many other parameters are believed to contribute to bubble formation including viscosity, surface tension, hydrated ion size, power input, and possibly others. For the experiments reported here, free-stream velocity (wall shear rate), injector pore diameter, and air injection flow rate were held constant to clearly extract the effects of salt and surfactant concentrations. The results presented here are intended to motivate additional research on bubble formation in turbulent shear flows, and to aid the comparison and interpretation of bubble-based skin-friction drag reduction tests in fresh, salt, and surfactant-laden water.

\section{2}

\section{Experimental methods}

The main experimental platform was a water tunnel with a $0.23 \mathrm{~m} \times 0.23 \mathrm{~m}$ test section, and the primary experimental measurements were photographic images processed manually to extract bubble-size distributions. The basic experimental variable was the water's concentration of solute-either salt or surfactant. The tunnel contained a water mass of $3,900 \pm 100 \mathrm{~kg}$ (corresponding volume of $3.9 \pm 0.1 \mathrm{~m}^{3}$ or $1,030 \pm 30$ gal) as determined from solid geometry, and verified with conductivity tests after known quantities of salt were added to the tunnel water. Before each test, the water tunnel was run for a minimum of $10 \mathrm{~min}$ to ensure uniform distribution of the solute. During this mixing period, the tunnel water cycled through the test section approximately 100 times.

The bubble injector was located on the upper test section window $0.27 \mathrm{~m}$ downstream from the exit of the 9:1 axisymmetric contraction. The bubble injector consisted of a $40 \mu \mathrm{m}$ mean-pore-diameter sintered metal plate (Madavan et al. 1984b) spanning $60 \mathrm{~mm}$ in the cross-stream direction and $20 \mathrm{~mm}$ in the stream-wise direction. Three layers of baffles with $500 \mu \mathrm{m}$ diameter holes were located between the air supply line and the sintered metal plate to distribute the air-flow evenly along the injector. A schematic of the injector is shown in Fig. 1.

For all tests the free-stream velocity in the test section was $16 \mathrm{~m} / \mathrm{s}$ and the air-flow rate was $85 \pm 3$ standard liters per minute (3.0 $\pm 0.1 \mathrm{SCFM})$, corresponding to $23.6 \pm 0.8$ standard liters per second per meter of span. An LDV survey of the wall boundary layer at the air injection location (without air injection) produced the mean velocity profile shown in Fig. 2. A 1/7th power-law velocity profile of the form

$\frac{u(y)}{U_{\infty}}=\left(\frac{y}{\delta_{99}}\right)^{1 / 7}$

was fitted to the measured profile. Here $y$ is the distance from the wall and $U_{\infty}$ is the free-stream velocity. 


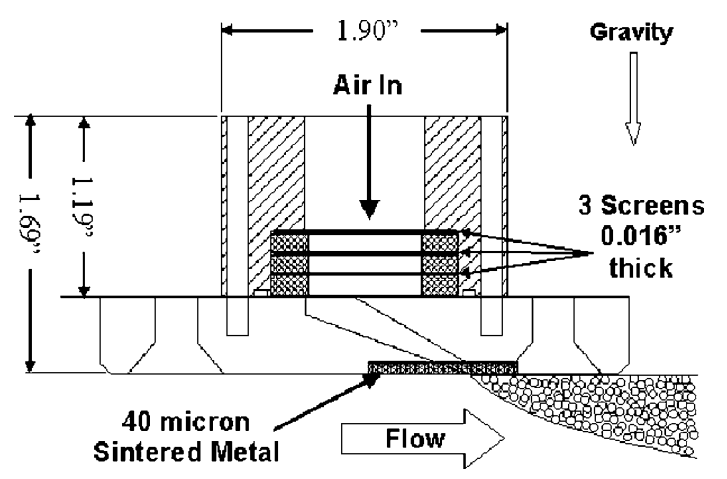

Fig. 1. Cross-sectional schematic of the air injector. Three brass screens with $500 \mu \mathrm{m}$ diameter holes serve to distribute evenly the air across the injector. The slot has a $10^{\circ}$ taper to accelerate the air-flow prior to passage through the porous metal and into the liquid boundary layer. The bubbles depicted are to help clarify the injection mechanism, but are not representative of the bubble size or a particular void fraction

The $99 \%$-boundary layer thickness, $\delta_{99}$, was determined from the experimental data to be $4.3 \mathrm{~mm}$ at the injection location. The corresponding boundary layer displacement thickness, $\delta^{*}$, was inferred to be $0.54 \mathrm{~mm}$ from the $1 / 7$ th power-law fit to the data. These results along with the air injection rate yield the void fraction within the TBL, $Q_{a} /$ $\left(Q_{\mathrm{a}}+Q_{\mathrm{w}}\right)$, as $29 \pm 1 \% . Q_{\mathrm{a}}$ is the volumetric air-flow rate and $Q_{w}$ is the water flow rate in the boundary layer defined by $Q_{\mathrm{w}}=U_{\infty}\left(\delta_{99}-\delta^{*}\right) b$ where $b$ is the injector span (Madavan et al. 1984a). The chosen volumetric flow rate of air per unit span matched that obtained in recent large-scale bubble drag reduction experiments (Sanders et al. 2004). Unfortunately, the test section pressure was not constant, and increased from 130 to $150 \mathrm{kPa}$ (absolute) during a test. However, from the ideal gas law, this pressure increase should only decrease bubble diameters by $4.6 \%$ and consequently was ignored in the data analysis. During these tests water temperature ranged from 24.0 to $26.5^{\circ} \mathrm{C}$.

Experiments were performed in freshwater (tap water), at four different concentrations of saltwater (Instant Ocean, Aquarium Systems), and at three different concentrations of a soluble surfactant (Triton-X-100,

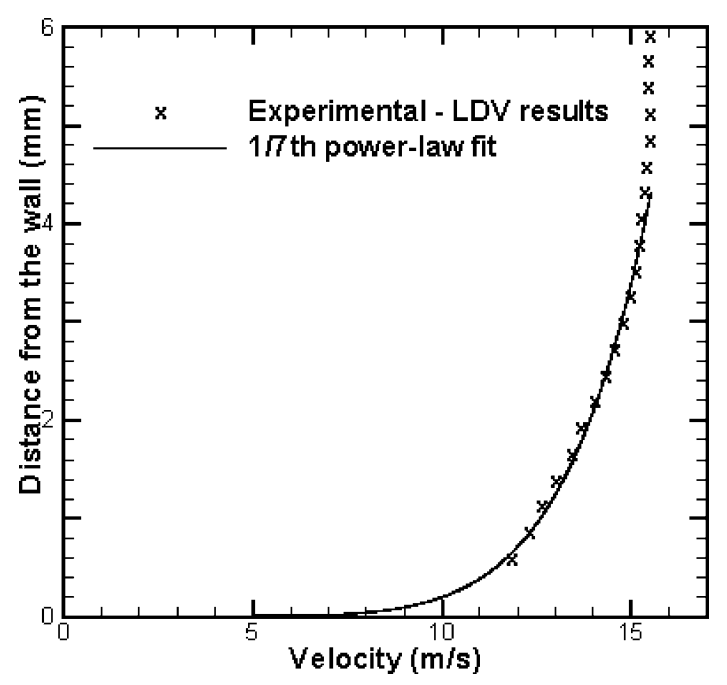

Fig. 2. Wall boundary layer profile at location of air injection
Union Carbide). Saltwater concentrations of 9, 19, 33 and 38 parts per thousand (ppt) were investigated, each accurate to within $\pm 0.3 \mathrm{ppt}$. These concentrations were determined by measuring the specific gravity and conductance of water samples taken from the tunnel followed by comparisons to previously generated calibration curves. The uncertainty is due to slight deviations in conductance of the samples tested. Specific conductance was measured using a Cole Parmer 1481-01 conductivity meter and the measured conductance values for each test condition are given in Table 1. The tested solutions of the soluble surfactant, 1,5 and $20 \mathrm{ppm}$, were obtained by adding 3.9, 19.0, and $76.8 \mathrm{~g}$ of Triton-X-100 to the tunnel water volume. This surfactant is safe, easy to obtain, and a reasonable stand-in for naturally occurring ocean surfactants (Dr. E. Bock, 1994, personal communication). The chosen surfactant concentrations should comfortably span naturally occurring surface tension variations.

Surface tension measurements were conducted on water samples from each experimental condition and are provided in Table 1 . Tensiometry measurements were made using the equipment and procedures described in Lapham et al. (1999). The surface tension results reported here are slightly lower than previous measurements at these concentrations using the same apparatus (Lapham et al. 2001). The lower surface tension measurements are likely due to less stringent laboratory hygiene, and because the tensiometry measurements in the current tests were not conducted until approximately $1 \mathrm{~h}$ after mixing. Triton-X-100 solutions are known to have time-dependent surface properties (Lapham et al. 2001).

Photographic images of the near-wall bubbles produced by the injector were recorded on ASA 800 film with 60 lines per millimeter resolution, using a Nikon $8008 \mathrm{~S}$ $35 \mathrm{~mm}$ camera that looked downward into the test section immediately downstream of the injector. The laboratory was darkened and the camera shutter was left open while the film was exposed using two synchronized strobe lights located above the test section window to illuminate the bubbly layer. The lighting, camera, and injector setup are shown in Fig. 3. The camera viewing area began $55 \mathrm{~mm}$ from the downstream edge of the injector plate, and spanned $15 \mathrm{~mm}$ in the stream-wise direction and $25 \mathrm{~mm}$ in the cross-stream direction. This viewing area is also shown in Fig. 3. At the given air-flow rate, the skin-friction drag reduction that could be obtained at this location would be $50 \%$ based on the results of Madavan et al. (1985) (skin-friction measurements were not made as part of the current study). The camera was focused on the window-water interface and the lens provided a depth of field of approximately $2 \mathrm{~mm}$ into the bubbly TBL flow.

For all conditions, photographs were recorded during and after air injection to ensure that the bubbles being photographed were those produced directly from the injector and not from recirculated air in the free stream. At the given test section speed, the recirculation time is estimated to be approximately $6 \mathrm{~s}$. Although many of the photographs were taken at multiple recirculation times after the first injection, these photographs were observed to be fundamentally no different than those taken immediately after injection started. Therefore, free-stream bubbles 
Table 1. Predicted ionic strength and specific conductance and surface tension measurements

\begin{tabular}{lccc}
\hline Solution & Ionic strength $(\mathrm{M})$ & $\begin{array}{l}\text { Spec. conductance } \\
(\mathrm{mS} / \mathrm{cm})\end{array}$ & $\begin{array}{c}\text { Surface tension } \\
(\text { dyne/cm })\end{array}$ \\
\hline Baseline (tap water) & $\sim 0.01$ & $0.50 \pm 0.05$ & $67.3 \pm 0.8$ \\
Surfactant (1 ppm) & $\mathrm{n} / \mathrm{a}$ & $0.48 \pm 0.05$ & $56.8 \pm 0.3$ \\
Surfactant (5 ppm) & $\mathrm{n} / \mathrm{a}$ & $0.48 \pm 0.05$ & $43.8 \pm 1.0$ \\
Surfactant (20 ppm) & $\mathrm{n} / \mathrm{a}$ & $0.49 \pm 0.05$ & $39.5 \pm 0.6$ \\
Instant Ocean (9 ppt) & $0.153 \pm 0.005$ & $8.1 \pm 0.2$ & $68.2 \pm 0.2$ \\
Instant Ocean (19 ppt) & $0.322 \pm 0.005$ & $16.5 \pm 0.2$ & $68.8 \pm 0.2$ \\
Instant Ocean (33 ppt) & $0.560 \pm 0.005$ & $27.1 \pm 0.2$ & $69.2 \pm 0.03$ \\
Instant Ocean (38 ppt) & $0.645 \pm 0.005$ & $30.7 \pm 0.3$ & $69.3 \pm 0.03$ \\
\hline
\end{tabular}

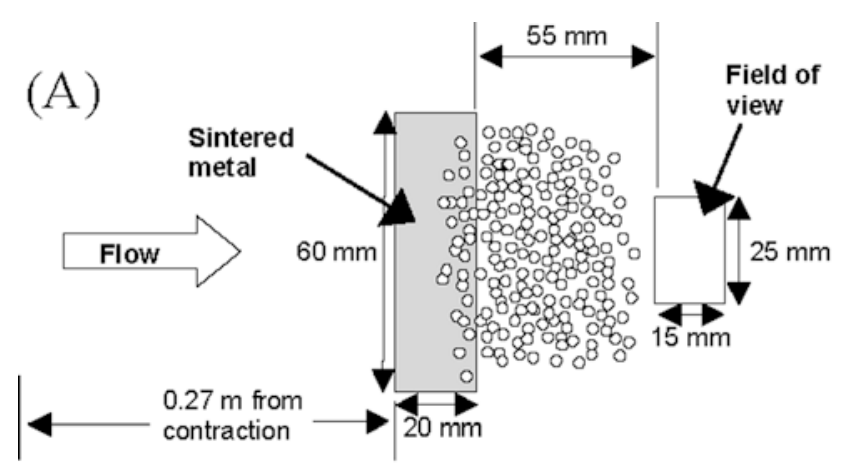

(B)

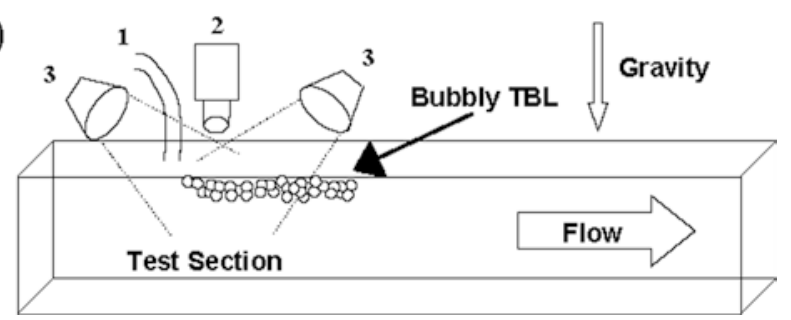

Fig. 3. Schematic diagrams of experimental setup. a Planform view from inside the test section of the sintered metal plate and camera viewing area. b Isometric view of the tunnel test section where 1,2, and 3 represent the injector air line, $35 \mathrm{~mm}$ camera, and strobe lights, respectively. Note: the bubbles depicted are not intended to represent size distribution or void fraction in the boundary layer

from the early portion of a test had little or no effect on the near wall bubbles just downstream of the injector.

The photographs were digitized and analyzed using Video Savant software (version 3.0) to quantify the bubble-size distribution. Line segments were fitted by eye across the diameter of all the distinguishable bubbles in each photograph. Bubbles without clearly defined edges were not included in the statistical analysis. The fitted line segments constitute the second image shown in Fig. 4.
Video Savant's blob analysis tool was used to determine the pixel length of each segment. The measured pixel image ratio $(1$ pixel $=29 \mu \mathrm{m})$ then allowed each bubble pixel length to be converted into a physical length. With the magnification of the camera lens, the resolution of the photographs, $12 \mu \mathrm{m}$, was slightly smaller than the digitized images. However, visual inspection did not reveal the presence of bubbles smaller than the $29 \mu \mathrm{m}$ digitized resolution. A minimum of 750 bubble diameters was measured at each flow condition. For bubble-volume calculations, all bubbles were treated as being spherical even though some were slightly elliptical. In addition, the bubble photographs only portray the bubbles in two dimensions. Here circular photographic bubble shapes were assumed to come from spherical bubbles. The bubble-volume error associated with these approximations is believed to be less than the bubble-volume uncertainty introduced by the variation in tunnel pressure.

\section{3}

\section{Results}

The bubble-size experiments for salt and the soluble surfactant were conducted separately; their combined effects were not investigated. Sample photographs of the bubble populations taken near the wall are given for all the conditions tested in Fig. 5. They clearly show that as the solute concentrations increase, the bubble populations are smaller in size, and are also more evenly distributed over the viewing area.

Measured bubble-size histograms, normalized so that they can be interpreted as probability density functions (PDFs), are shown in Fig. 6, for salt and the soluble surfactant solutions, respectively. Table 2 summarizes the mean bubble-diameter and bubble-volume results. When $l_{v}$ is estimated from standard empirical correlations, $d^{+}$is of the order $10^{2}$ for all the measured conditions. Here, the mean bubble diameter was calculated
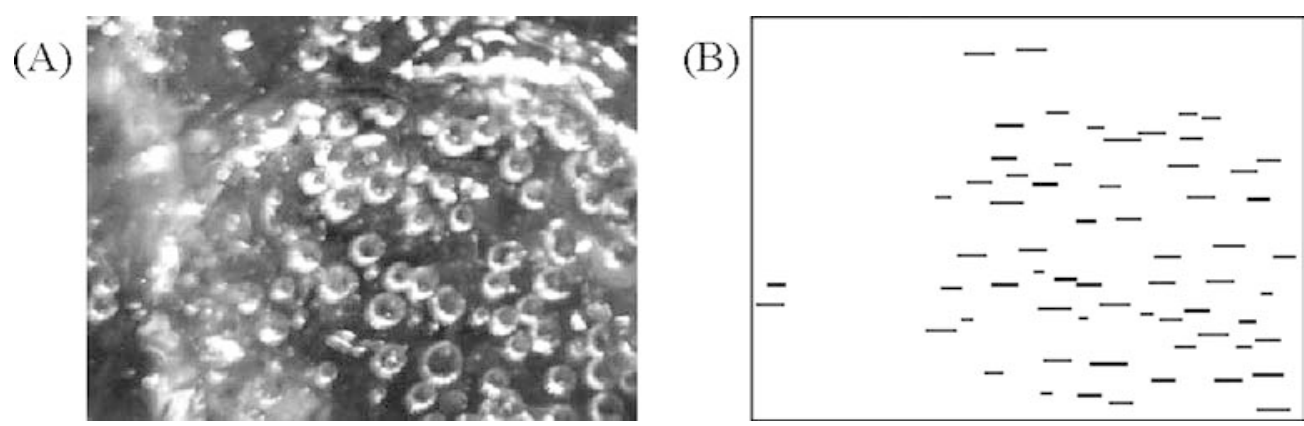

Fig. 4. Bubble measurement technique. a Photograph of bubbly layer. b Line segments manually fitted across bubble diameters. Note: this is only one fourth of the total viewing area, and the photograph is from the $5 \mathrm{ppm}$ surfactant condition 


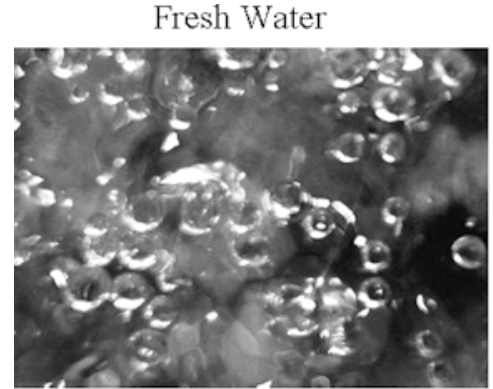

806

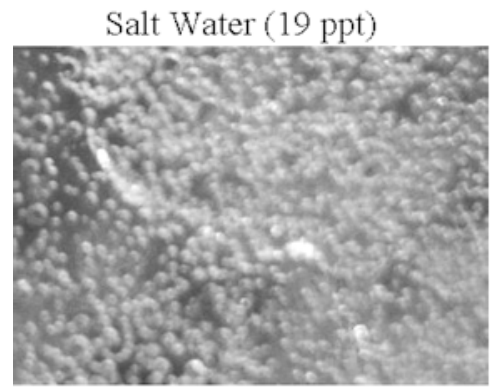

Salt Water (38 ppt)

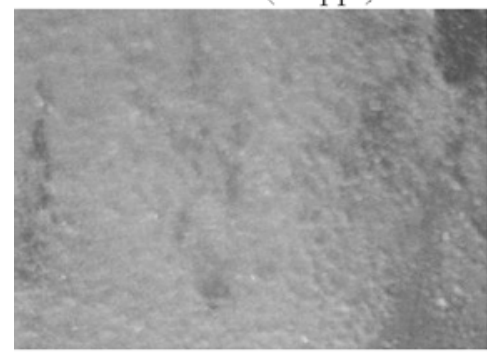

Surfactant (5 ppm)

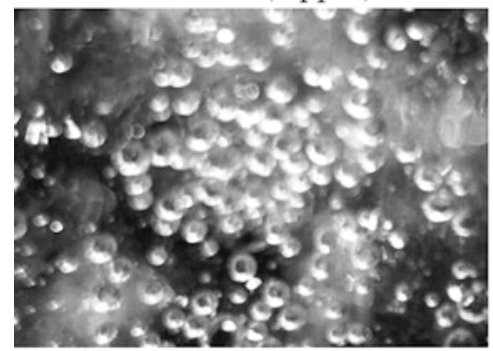

Salt Water (9 ppt)

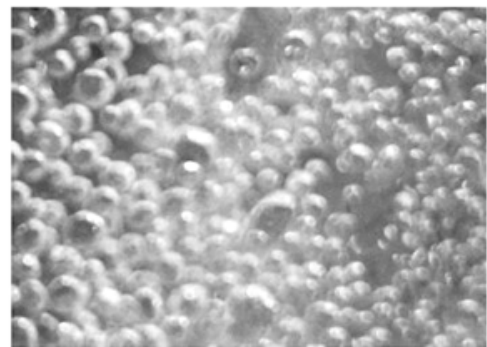

Salt Water (33 ppt)

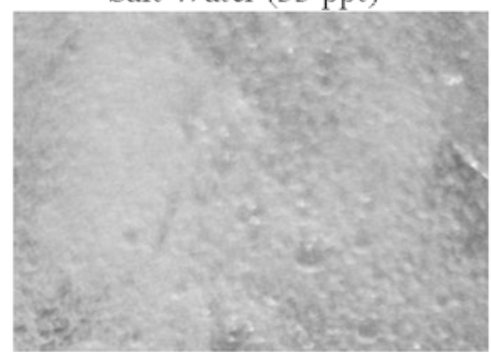

Surfactant (1 ppm)

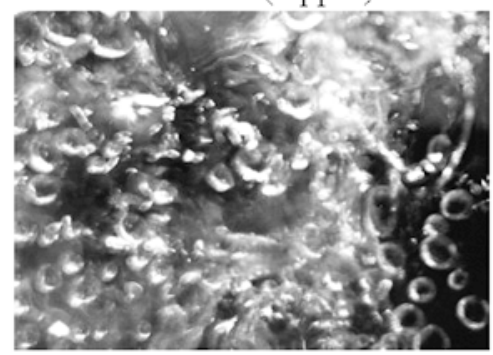

Surfactant (20 ppm)

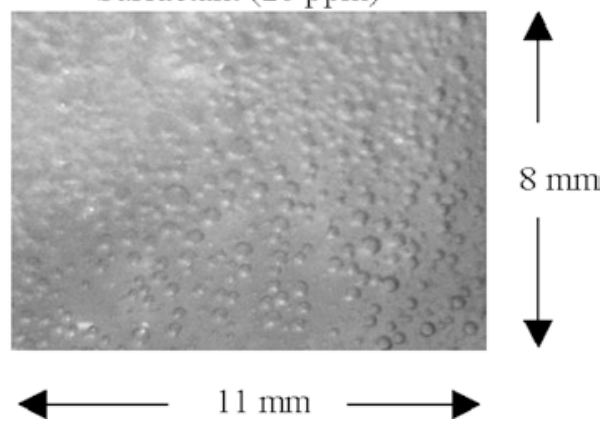

Fig. 5. Sample photographs for all the bubble injection conditions tested. These photographs do not show the entire $15 \mathrm{~mm} \times 25 \mathrm{~mm}$ viewing area, but an $8 \mathrm{~mm} \times 11 \mathrm{~mm}$ section. In all the photographs, the flow is toward the top of the page from the tabulated bubble-diameter measurements by summing and dividing by the number of measurements. The uncertainties reported for the mean bubble diameters are assumed to be twice the standard error of the mean. The mean bubble volume was computed by assuming the bubbles were spherical, and then summing bubble volumes and dividing by the number of measurements. In both solutions, bubble sizes decreased monotonically with increasing solute concentration. The two largest saltwater concentrations ( 33 and $38 \mathrm{ppt}$ ) decrease the freshwater mean bubble diameter by more than a factor of four while the highest surfactant concentration lowered it by more than a factor of two.

In addition to these directly tabulated experimental results, lognormal fits to the experimental histograms were constructed by presuming $\ln (d)$ to be normally distributed and calculating its mean, $m$, and variance $s^{2}$ directly from the tabulated bubble diameters. Here the fitted probability density function, $P(d)$, is defined by

$P(d)=\frac{\exp \left[-\frac{1}{2}\left(\frac{\ln (d)-m}{s}\right)^{2}\right]}{s d \sqrt{2 \pi}}$

and the results for $m$ and $s$ are shown in Table 3 for each condition. The uncertainties reported in $m$ and $s$ are a direct result of the natural variability in bubble diameters. The most probable bubble diameters have been inferred from the fitted lognormal PDFs and are given in Table 2. The corresponding lognormal PDFs are shown in Fig. 6 along with the tabulated histograms.

Overall, the principal factor responsible for changes in bubble formation appear to be different for the saltwater and surfactant solutions. The bulk properties of saltwater 

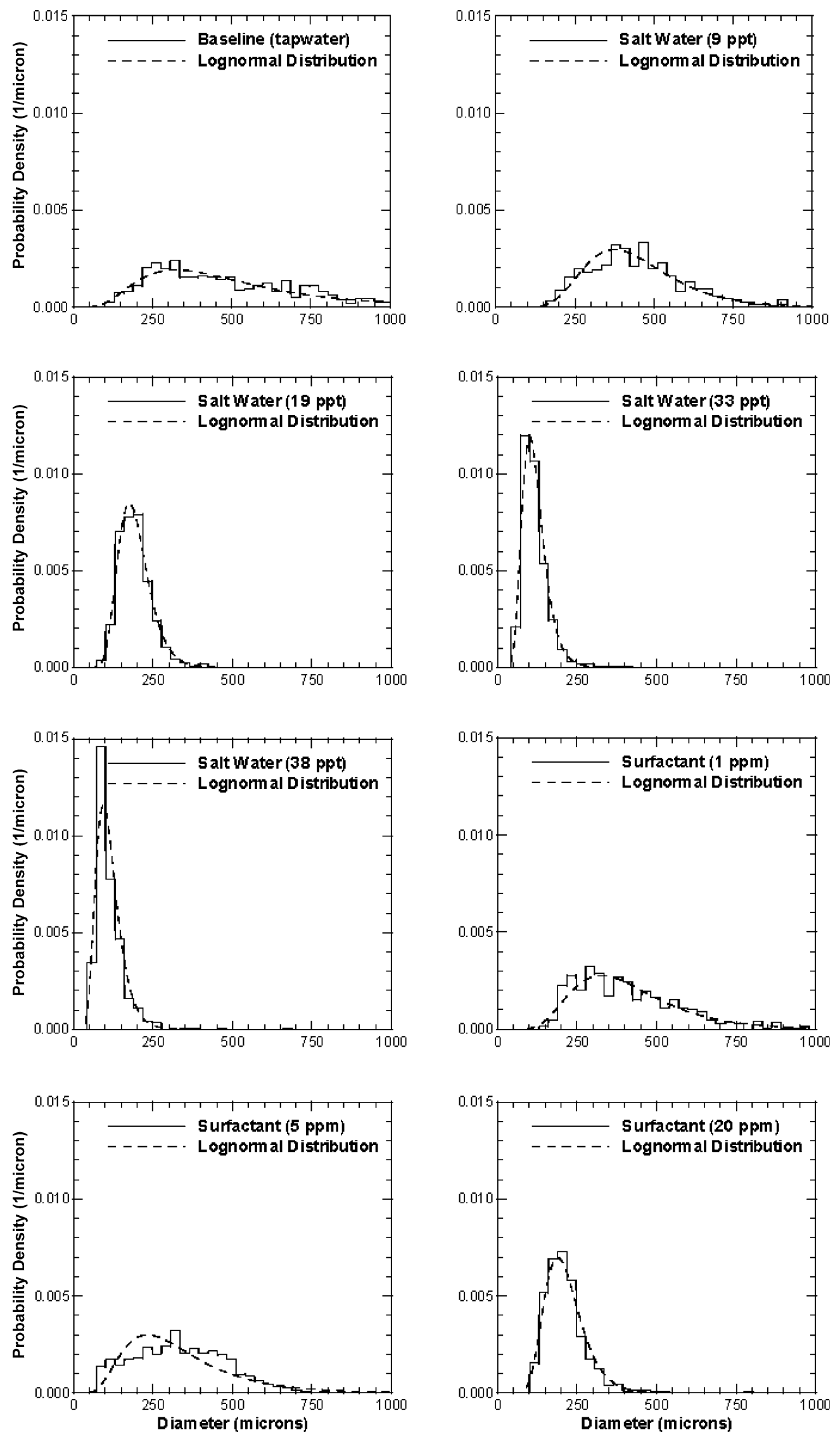

Fig. 6. Normalized histograms and lognormal PDFs of tabulated bubble distributions for saltwater and surfactant solutions

are only marginally different from freshwater, $2.5 \%$ more change that determines variations in bubble-size dense, $7 \%$ more viscous, and $2 \%$ increased surface tension distribution in the saltwater may be the increased ionic (Slauenwhite and Johnson 1999). Therefore, the dominant strength of the solution, given in Table 1. Fig. 7 shows the 
Table 2. Mean bubble diameters, mean bubble volumes, most probable diameters, and $d^{+}$values

\begin{tabular}{lcccc}
\hline Solution & $\begin{array}{c}\text { Mean bubble } \\
\text { diameter }(\mu \mathrm{m})\end{array}$ & $\begin{array}{l}\text { Mean bubble } \\
\text { volume }\left(\mathrm{mm}^{3}\right)\end{array}$ & $\begin{array}{c}\text { Most probable } \\
\text { diameter }(\mu \mathrm{m})\end{array}$ & $d^{+}=d / l_{v}$ \\
\hline Baseline (tap water) & $491 \pm 18$ & $0.109 \pm 0.063$ & $435 \pm 10$ & $323 \pm 12$ \\
Surfactant (1 ppm) & $420 \pm 17$ & $0.066 \pm 0.024$ & $385 \pm 9$ & $276 \pm 11$ \\
Surfactant (5 ppm) & $337 \pm 7$ & $0.032 \pm 0.029$ & $301 \pm 4$ & $221 \pm 5$ \\
Surfactant (20 ppm) & $215 \pm 4$ & $0.007 \pm 0.003$ & $205 \pm 2$ & $141 \pm 3$ \\
Instant Ocean (9 ppt) & $446 \pm 12$ & $0.063 \pm 0.022$ & $421 \pm 6$ & $293 \pm 8$ \\
Instant Ocean (19 ppt) & $194 \pm 3$ & $0.0047 \pm 0.0013$ & $187 \pm 1$ & $127 \pm 2$ \\
Instant Ocean (33 ppt) & $118 \pm 3$ & $0.0012 \pm 0.0003$ & $112 \pm 1$ & $78 \pm 2$ \\
Instant Ocean (38 ppt) & $113 \pm 3$ & $0.0011 \pm 0.0005$ & $105 \pm 2$ & $74 \pm 2$ \\
\hline
\end{tabular}

Table 3. Lognormal probability density function coefficients $m$ and $s$ for bubble diameter distributions

\begin{tabular}{lll}
\hline Solution & $m$ & $s$ \\
\hline Baseline (tap water) & $6.075 \pm 0.023$ & $0.502 \pm 0.012$ \\
Surfactant (1 ppm) & $5.953 \pm 0.023$ & $0.408 \pm 0.009$ \\
Surfactant (5 ppm) & $5.707 \pm 0.013$ & $0.503 \pm 0.08$ \\
Surfactant (20 ppm) & $5.324 \pm 0.010$ & $0.288 \pm 0.003$ \\
Instant Ocean (9 ppt) & $6.043 \pm 0.015$ & $0.339 \pm 0.005$ \\
Instant Ocean (19 ppt) & $5.233 \pm 0.008$ & $0.259 \pm 0.002$ \\
Instant Ocean (33 ppt) & $4.717 \pm 0.010$ & $0.315 \pm 0.003$ \\
Instant Ocean (38 ppt) & $4.661 \pm 0.014$ & $0.346 \pm 0.004$ \\
\hline
\end{tabular}

mean bubble diameters for the saltwater solutions plotted with the ionic strength, $I$, of the solution, defined by

$I=\frac{1}{2} \sum_{i=1}^{k}\left(m_{i} Z_{i}^{2}\right)$

where $m_{i}$ is the molality of ion $i$, and and $Z_{i}$ is the charge of the ion (Fried et al. 1977). The major ionic species present in the Instant Ocean synthetic sea salt are given in Table 4. Accordingly, the interfacial surface area per unit volume of injected air increases monotonically with increasing ionic strength (from 0.01 to $0.66 \mathrm{M}$ ), shown in Fig. 8. These results agree qualitatively with those found by Zieminski and Whittemore (1971), who dispersed air through a
Table 4. Major constituents of Instant Ocean Synthetic Sea Salt (adapted from TAF-REV-1023-91 Aquarium Systems, 8141 Tyler Boulevard, Mentor, Ohio)

\begin{tabular}{lcl}
\hline \multicolumn{2}{l}{ Ions/constituents } & Weight (\%) \\
\hline Chlorine & $\mathrm{Cl}^{-}$ & 47.47 \\
Sodium & $\mathrm{Na}^{+}$ & 26.28 \\
Sulfate & $\mathrm{SO}_{4}{ }^{2-}$ & 6.602 \\
Magnesium & $\mathrm{Mg}^{2+}$ & 3.23 \\
Calcium & $\mathrm{Ca}^{2+}$ & 1.013 \\
Potassium & $\mathrm{K}^{+}$ & 1.015 \\
Bicarbonate & $\mathrm{HCO}_{3}^{-}$ & 0.491 \\
Boron & $\mathrm{B}^{2+}$ & 0.015 \\
Strontium & $\mathrm{Sr}^{2+}$ & 0.001 \\
Water & $\mathrm{H}_{2} \mathrm{O}$ & 13.88 \\
Total & & 99.99 \\
\hline
\end{tabular}

porous plate into a quiescent bath of solution. Their results show a linear relationship between interfacial area and ionic strength (from 0.00 to $0.45 \mathrm{M}$ ). One of the physical mechanisms for the decrease in bubble size is presumed to be the ionic repulsion between bubbles in saltwater that prevents bubble coalescence near the injector (Slauenwhite and Johnson 1999).

Figure 9 shows mean bubble diameter results for the surfactant solutions, with higher surfactant concentrations producing lower surface tension solutions. Here, the

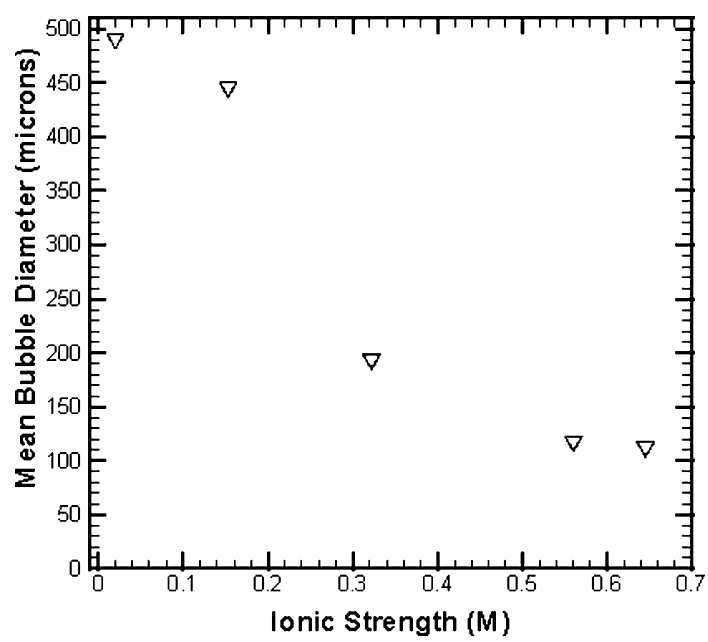

Fig. 7. Relationship between ionic strength and mean bubble diameter for the saltwater solutions

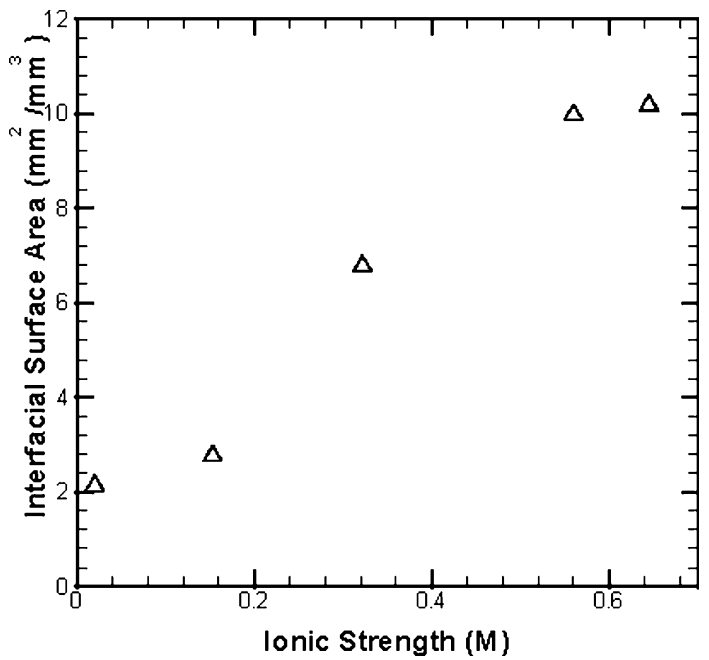

Fig. 8. Normalized interfacial surface area vs. ionic strength for the saltwater solutions 


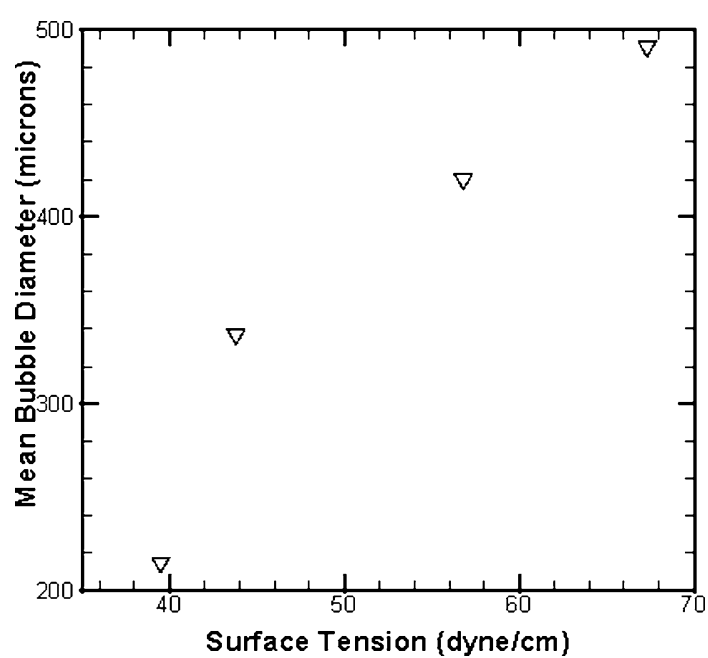

Fig. 9. Relationship between surface tension and mean bubble diameter for the surfactant solutions

measured mean bubble diameter decreases with decreasing surface tension. This diameter change can most likely be attributed to the drop in surface tension. Using $\sigma$ as the surface tension, $n$ as the number density of bubbles, and $d$ as a representative bubble diameter, the bubble-surface energy per unit volume of flow will be proportional to $n \sigma \mathrm{d}^{2}$ while the volume of air per unit volume of flow will be $n d^{3}$. If the bubble-surface energy per unit volume of injected air is independent of surface tension, then $n \sigma \mathrm{d}^{2} / n d^{3}=\sigma / d$ should be constant. This trend is closely followed by the present bubbly surfactant-solution measurements for surface tension values between 44 and 67 dyne $/ \mathrm{cm}$. However, other phenomena appear to be active at lower surface tension since unexpectedly small bubbles are observed at a surface tension of 39.5 dyne $/ \mathrm{cm}$ (20 ppm Triton-X-100) when compared to the other three data points in Fig. 9.

\section{4}

\section{Conclusions}

The data presented here lead to the following three conclusions.

1. The bubbles produced by air injection through porous metal into a relatively high-speed turbulent boundary layer are significantly different in saltwater and freshwater. The observations that support this conclusion were made approximately $6 \mathrm{~cm}$ downstream of the air injector, so it is not possible to state definitively whether near-injector bubble coalescence or the bubble formation process, or both, are altered by the presence of dissolved salt. However, the combined phenomena must be different because the mean bubble diameter was reduced from its freshwater value by more than a factor of four for salt concentrations similar to that of seawater.

2. The bubble-size reduction obtained with seawater-level salt concentrations was not reached with lower concentrations of salt or with a range of surfactant concentrations. Thus, the effect of aqueous ions on bubble size does not saturate at low salt concentrations, and the observed salt-induced bubble-size reduction cannot be readily mimicked by lowering the water's surface tension with a soluble surfactant. This means it may not be possible to reproduce the effects of seawater in freshwater test facilities by using less-corrosive salt or surfactant solutions.

3 Although this study is limited in scope, it shows that changes in bubble size could be a significant difference between bubble drag reduction effectiveness in saltwater and freshwater. Only one flow speed, one air injector, and one air injection rate were tested; however, these results should motivate further investigation into the effects of saltwater and surfactants on bubble formation and bubble-induced skin-friction drag reduction.

\section{References}

Alves S, Maia C, Vasconcelos J, Serralheiro A (2002) Bubble size in aerated stirred tanks. Chem Eng J 89:109-117

Bogdevich V, Evseev A (1976) The distribution of skin-friction in a turbulent boundary layer of water beyond the location of gas injection. In: Migirenko GS (ed) Investigations of boundary layer control. Thermophysics Institute Publishing, Novosibirsk

Fried V, Hameka HF, Bulkis U (1977) Physical chemistry. MacMillan, New York

Kato H, Fuji Y, Yamaguchi H, Miyanaga M (1993) Frictional drag reduction by injecting high-viscosity fluid into a turbulent boundary layer. J Fluids Eng 115:206-212

Kawamura T, Moriguchi Y, Kato H, Kakugawa A, Kodama Y (2003) Effect of bubble size on the microbubble drag reduction of a turbulent boundary layer. ASME joint fluids engineering conference

Keitel G, Onken U (1982) Inhibition of bubble coalescence by solutes in air/water dispersions. Chem Eng Sci 37:1635-1638

Kitagawa A, Sugiyama K, Ashihara M, Hishida K, Kodama Y (2003) Measurement of turbulence modification by microbubbles causing frictional drag reduction. In: Proceedings of ASME fluids engineering division summer meeting, $\mathrm{pp} 1-7$

Kunz R, Deutsch S, Landau J (2003) Two fluid modeling of microbubble turbulent drag reduction. In: Proceedings of ASME fluids engineering division summer meeting, pp 1-10

Lapham G, Dowling D, Schultz W (1999) In situ force-balance tensiometry. Exp Fluids 27:157-166

Lapham G, Dowling D, Schultz W (2001) Linear and nonlinear gravity-capillary water waves with a soluble surfactant. Exp Fluids 30:448-457

Legner H (1984) A simple model for gas bubble drag reduction. Phys Fluids 27:2788-2790

Lumley J (1977) Drag reduction in two phase and polymer flows. Phys Fluids 20:S64-S70

Machon V, Pacek A, Nienow A (1997) Bubble sizes in electrolyte and alcohol solutions in turbulent stirred vessel. Trans Ind Chem Eng 75A:339-347

Madavan N, Deutsch S, Merkle C (1984a) Reduction of turbulent skinfriction by microbubbles. Phys Fluids 27:356-363

Madavan N, Deutsch S, Merkle C (1984b) The effect of porous material on microbubble skin-friction reduction. Am Inst Aeronaut Astronaut, paper no 84-0348

Madavan N, Deutsch S, Merkle C (1985) Measurments of local skinfriction in a microbubble-modified turbulent boundary layer. J Fluid Mech 156:237-256

Meng J, Lovett J (1998) Microbubble hydrodynamic drag reduction: High speed subscale experiment and estimated improvements in full-scale implementation. NUWC-NPT technical report 11-038:1-52

Meng J, Uhlman J (1998) Microbubble formation and splitting in a turbulent boundary layer for turbulence reduction. In: Proceedings of the international symposium on seawater drag reduction, pp 341-355

Merkle C, Deutsch S (1992) Microbubble drag reduction in liquid turbulent boundary layers, Part 1. Appl Mech Rev 45(3):103-127 
Sanders WC, Dowling DR, Perlin M, Ceccio SL (2004) Bubble friction drag reduction in a high Reynolds number flat plate turbulent boundary layer. J Fluid Mech (in press)

Schlichting H (1979) Boundary layer theory, 7th edn. McGraw-Hill, New York

Slauenwhite D, Johnson B (1999) Bubble shattering: Differences in bubble formation in fresh water and seawater. J Geophys Res 104(C2):3265-3275
Takahashi T, Kakugawa A, Nagaya S, Yanagihara T, Kodama Y (2001) Mechanisms and scale effects of skin-friction reduction by microbubbles. In: Proceedings of 2nd symposium on smart control of turbulence

Zieminski S, Whittemore R (1971) Behavior of gas bubbles in aqueous electrolyte solutions. Chem Eng Sci 26:509-520 